\title{
MHD STABILITY OF TOROIDAL PLASMA WITH MODULATED CURVATURE PLANAR MAGNETIC AXIS
}

\author{
H. M. RuzK* \\ The university of Michigan, 1463-1222 University Terrace, Ann Arbor, MI 48109, U.S.A.
}

(Received 3 September 1979; and in revised form 20 August 1980)

\begin{abstract}
Mercier's localized perturbed criterion of stability near an arbitrary modulated curvature magnetic axis of a non-circular toroidal plasma cross section is investigated. In this magnetic configuration, the magnetic surfaces arbitrarily rotate around the magnetic axis. The influence of the non-circular cross section of the magnetic surface and that of the modulation of the magnetic axis on the domains of equilibrium and stability are studied.
\end{abstract}

\section{INTRODUCTION}

MERCIER's general geometric criterion of stability for a magnetic toroical configuration of a modulated curvature magnetic axis and circular plasma cross section is calculated by Shafranov (1968). Adams and Mercier (1969) and Mikhamov. SKII and ABURDZHANTYA $(1978,1979)$, but with a different method from that used here.

In the present work, we study the equilibrium and analyze Mercier's criterion of stability (MERCIER, 1964) near an arbitrary modulated curvature planar magnetic axis (with zero torsion and variable curvature) of non-circular (elliptically and triangularly deformed) toroidal plasma cross section with high pressure. A longitudinal uniform current is allowed to flow through the plasma. Also, we study the influence of the modulation of the magnetic axis on the equilibrium and stability of this plasma configuration.

By introducing the coordinates $(\rho, \theta, s)$ (see Fig. 1), where $(\rho, \theta)$ is the polar coordinate and $s$ is the curvilinear coordinate. The curvature of modulated curvature planar magnetic axis (hereafter referred to as m.a.) is represented by $(1 / R(s))=\sum_{k=-\infty}^{+\infty} a_{k} \exp (2 \pi k i s / L)$ where $a_{-k}=a_{+k}$ and $L=\oint \mathrm{d} s$ is the total length of the magnetic axis. If all the $k$ 's are even, this curve will be closed with $a_{0}=2 \pi / L$. The simplest form for the closed modulated curvature planar magnetic axis is given by:

$$
\frac{1}{R(s)}=a_{0}\left(1+2 \mu_{k} \cos \frac{2 \pi k s}{L}\right)
$$

where $\mu_{k}=a_{k} / a_{0}$ is the depth of modulation and $a_{0}=2 \pi / L$.

At this point, one would like to mention that the magnetic surfaces rotate $-k$ ( $=$ the number of modulation periods or the resonance index related to the resonant Fourier coefficient $a_{k}$ ) times around the magnetic axis (Luc et al. 1974). This rotation $\mathrm{d}^{\prime}(s) / 2(=2 \pi k / L)$ which is associated with the non-vanishing longitudinal current $I_{s}$, is necessary in order to apply the method of helical images. Also, one can see from the equilibrium solution considered here, that this rotation is important when the magnetic surface chosen has a non-circular or circular (at

* Present address: 10 Josyln St., Newport, Maine 04953. U.S.A. 


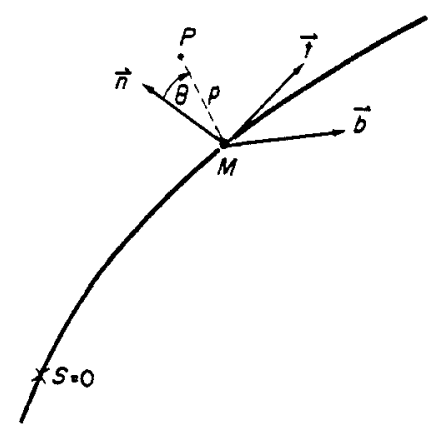

FIG. 1.-System of coordinates.

high pressure) cross section. It can be neglected at low pressure since the magnetic surfaces are nearly concentric circles. This toroidal plasma configuration is a geometric model which is only an image of the real configuration. Moreover, the MHD equilibria of the Tokamak is just a special case of the equilibrium solution given here.

At the Fusion Laboratory of Fontenay-aux-Roses in France, a toroidal plasma configuration with modulated curvature magnetic axis with $k=2$, called 'Harmonica-II' was built (see Fig. 2). This experiment successfully demonstrated Mercier's result (MERCIER, 1964).

\section{EQUILIBRIUM}

The equations of interest are the ideal MHD equations: $\boldsymbol{\nabla} \cdot \mathbf{B}=0, \boldsymbol{\nabla} \times \mathbf{B}=\mathbf{j}$ and $\mathbf{j} \times \mathbf{B}=\nabla P$ where $\mathbf{B}, \mathbf{j}$, and $P$ are respectively the magnetic field, current density, and scalar pressure.

The expressions for the magnetic field, current density, and the MHD equilibrium equation can be obtained with the aid of the helical images hypothesis by putting $1 / T=0, \theta=\theta_{0}$, and $\varepsilon_{t}=\varepsilon_{m}$ in the set of equations given in reference (LuC et al., 1974). The result is:

$$
\begin{gathered}
\mathbf{B}=f \mathbf{u}+\mathbf{u} \wedge \nabla F \\
\mathbf{j}=\left(\mathscr{L}_{\text {op }} F+\frac{2 \varepsilon_{m} f}{a g}\right) \mathbf{u}+\mathbf{u} \wedge \nabla(-f) \\
\mathscr{L}_{\text {op }} F+\frac{2 \varepsilon_{m} f}{a g}+\frac{1}{2} \frac{\mathrm{d} f^{2}}{\mathrm{~d} F}+g \frac{\mathrm{d} P}{\mathrm{~d} F}=0
\end{gathered}
$$

FIG. 2.-Magnetic toroidal configuration with modulated curvature magnetic axis 'Harmonica-II'; $k=2$. 
where

$$
\begin{aligned}
g & =h_{k}^{2}+\left(\frac{\varepsilon_{m} \rho}{a}\right)^{2}, \quad h_{k}=\left(1-\frac{\varepsilon_{k} \rho}{a} \cos t\right), \quad t=\theta+\frac{\mathrm{d}(s)}{2}, \\
\mathbf{u} & =\frac{1}{g}\left(h_{\mathrm{k}} \mathbf{e}_{\mathrm{s}}+\frac{\varepsilon_{m} \rho}{a} \mathbf{e}_{\theta}\right), \quad \varepsilon_{k}=a a_{k}, \quad \varepsilon_{m}=-k \varepsilon_{0}, \\
\mathscr{L}_{\text {op }} & =\frac{g}{\rho h_{\mathrm{k}}}\left[\frac{\partial}{\partial \rho}\left(\frac{\rho h_{k}}{g} \frac{\partial}{\partial \rho}\right)+\frac{\partial}{\partial t}\left(\frac{1}{\rho h_{k}} \frac{\partial}{\partial t}\right)\right]
\end{aligned}
$$

$\varepsilon_{0}(=2 \pi a / L)$ is the inverse of the aspect ratio. $a$ is the characteristic dimension of the plasma cross section. $(d(s) / 2)(=2 \pi k s / L)$ is the turning angle of the minor axis of the ellipse near the m.a. The functions $f$ and $P$ are arbitrary functions of the equilibrium solution $F$.

We will choose $P_{(F)}=P_{0}+P_{1} F$ and $f_{(F)}^{2}=f_{0}{ }^{2}+\lambda F$ where $P_{0}, P_{1}, \lambda$ and $f_{0}$ are constant parameters. This respectively corresponds to a parabolic pressure profile and a nearly flat current density profile. The function $f$ has been extended to yield $f=f_{0}+O\left(\varepsilon^{2}\right)$. If we take $F=P=0$ on the plasma boundary. this leads to $P_{0}=0$. The analytical solution of equation (4) is given by:

$$
\begin{aligned}
F & =F_{s} F_{c} \\
F / F_{0} & =1-X^{2}-Y^{2}\left(\frac{1-E}{1+E}\right)-\varepsilon_{s}\left[X^{3}-3 X Y^{2}\left(\frac{1-E}{1+E}\right)\right] \\
F_{c} & =1-\nu X+\varepsilon_{k} \nu\left[\gamma_{1} X^{2}+\gamma_{2} Y^{2}\left(\frac{1-E}{1+E}\right)\right]
\end{aligned}
$$

$X=x / a, Y=y / a, x=\rho \cos t, y=\rho \sin t$, and $F_{0}, \nu, \gamma_{1}$, and $\gamma_{2}$ are constants. $\varepsilon_{s}$ is the appropriate distortion factor. $-1 \leq E \leq 1$ is the ellipticity parameter of the plasma boundary cross section. The equation of the triangular deformation cross section is given by: $F_{\mathrm{s}}=0$. Figure 3 shows the possible shapes of this plasma cross section. It has been assumed that $P_{(F)}=0, F=0$ on the toroidal surface of the

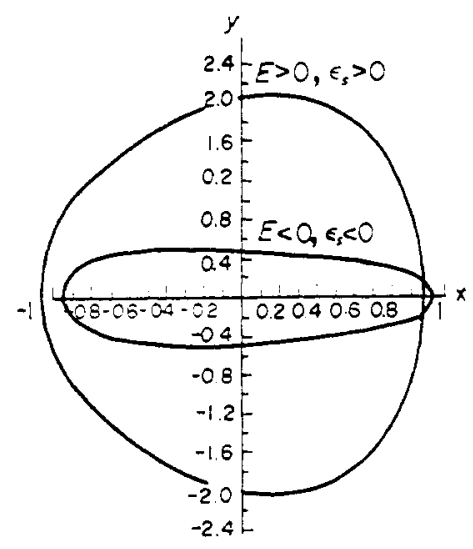

FIG. 3.-Possible shapes of the triangular deformation plasma cross section. 
non-circular cross section given by $F_{s}=0$. Also, we assume that $\varepsilon_{s} \sim \varepsilon_{k} \sim \varepsilon_{m} \sim$ $\varepsilon_{0} \ll 1$.

By introducing the parameters, the mean pressure

$$
\langle P\rangle=\frac{1}{V} \int P \mathrm{~d} V \simeq \frac{1}{2} P_{1} F_{0} ; \quad \beta=\frac{2\langle P\rangle}{\left\langle\left(B_{s}^{2}\right)_{X=1}\right\rangle} \simeq \frac{P_{1} F_{0}}{B_{0}^{2}},
$$

the inverse of the safety factor for an equivalent cylinder

$$
\frac{1}{q_{0}}=\frac{L}{2 \pi a} \frac{1}{\left(B_{\mathrm{s}}\right)_{\mathrm{X}=1}} \frac{I_{\mathrm{s}}}{2 \pi b} \cong \frac{a j_{0}}{2 \varepsilon_{0} B_{0}}(1+O(\varepsilon)) \text { and } G=-\left(\frac{1}{q_{0}}+k\right)
$$

where $B_{0}$ and $j_{0}$ are respectively the longitudinal magnetic field and current density at the plasma center, the expresions for $F_{0}, \nu, \gamma_{1}$, and $\gamma_{2}$ are given by:

$$
\begin{aligned}
& F_{0}=\frac{a \varepsilon_{0}}{2} B_{0}(1+E) G \\
& \frac{\nu}{\varepsilon_{k}}=\frac{1}{(1+E / 2)}\left\{\frac{1}{4}\left[1+\left(\frac{6 \varepsilon_{s}}{\varepsilon_{k}}+1\right) E\right]+\frac{k}{G}+\beta^{*}\right\} \\
& \gamma_{1}=\frac{1}{2}+\frac{4\left(\frac{\varepsilon_{s}}{\varepsilon_{k}}\right)\left(1+2 E-2 E^{2}\right)+\left(\frac{\varepsilon_{m}}{\varepsilon_{k}}\right)^{2}(1-E)(2+E)}{4\left(2-E^{2}\right)}
\end{aligned}
$$

and

where

$$
\gamma_{2}=\frac{(1+E)^{2}\left\{-\frac{4 \varepsilon_{s}}{\varepsilon_{k}}(1-E)+\left(\frac{\varepsilon_{m}}{\varepsilon_{k}}\right)^{2}(2+E)\right\}}{4\left(2-E^{2}\right)(1-E)}
$$

$$
\beta^{*}=\frac{\beta}{(1+E) \varepsilon_{0}^{2} G^{2}} .
$$

Figure 4 shows a typical plot of this magnetic surface.

The limiting value of $\beta$ for equilibrium corresponds to $\nu=1$ at which a new magnetic axis appears on the plasma boundary and is given

$$
\begin{aligned}
\beta_{P_{\max }} & =\varepsilon_{0}^{2} G^{2}(1+E)\left\{\frac{1}{\varepsilon_{k}}\left(1+\frac{E}{2}\right)-\frac{1}{4}\left[1+\left(\frac{6 \varepsilon_{s}}{\varepsilon_{k}}+1\right) E\right]-\frac{k}{G}\right\} \\
& =\left(\frac{\varepsilon_{0} G^{2}}{\mu_{k}}\right)(1+E)\left(1+\frac{E}{2}\right)(1+O(\varepsilon)) \sim \varepsilon .
\end{aligned}
$$

The investigation of this expression will be given in the stability section.

The expressions of the ratio $j_{s 0} / 2 B_{s 0}$ of the longitudinal current density $j_{s 0}$ and the longitudinal magnetic field $B_{s 0}$ near the m.a., the rotational transform $\iota_{c 0} / 2 \pi$ near the m.a., and the function $P_{0}^{\prime}$ (which is the derivative near the m.a. of the pressure with respect to the poloidal flux function) can be put in the following forms:

$$
\begin{gathered}
j_{s 0} / 2 B_{s 0}=\frac{\varepsilon_{0}}{a}\left(\frac{1}{q}+\beta^{*} G W\right) \\
\frac{\iota_{c 0}}{2 \pi}=-k+\frac{L \mathscr{K}}{2 \pi} \quad \text { with } \mathscr{K}=\sqrt{\left(1-E_{m}{ }^{2}\right)}\left(\frac{j_{s 0}}{2 B_{s 0}}+\frac{d^{\prime}(s)}{2}\right)
\end{gathered}
$$




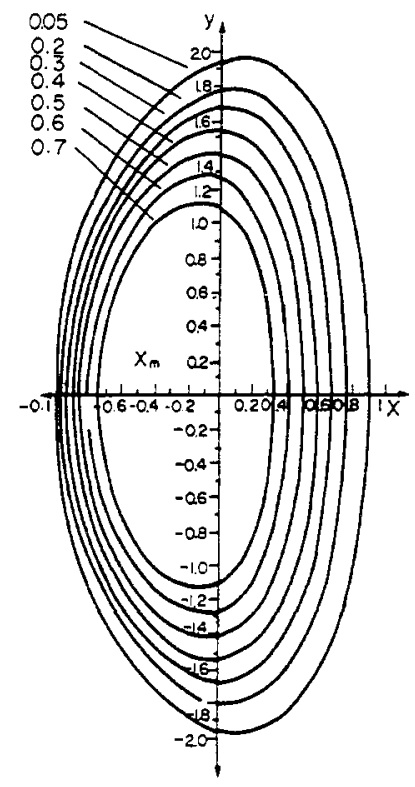

FIG. 4(a).-Magnetic surfaces in the plasma for $1 / q_{0}=0.2, \varepsilon_{s}=0.1, E=0.6, \varepsilon_{0}=0.1$, $\mu_{k}=1, k=2, \varepsilon_{k}=0.1, \alpha_{0}=0.024, \beta=0.57$, and different values of $F / F_{0}$. The magnetic axis is placed at distance $x_{m}=-0.246$ from the central axis of the plasma.

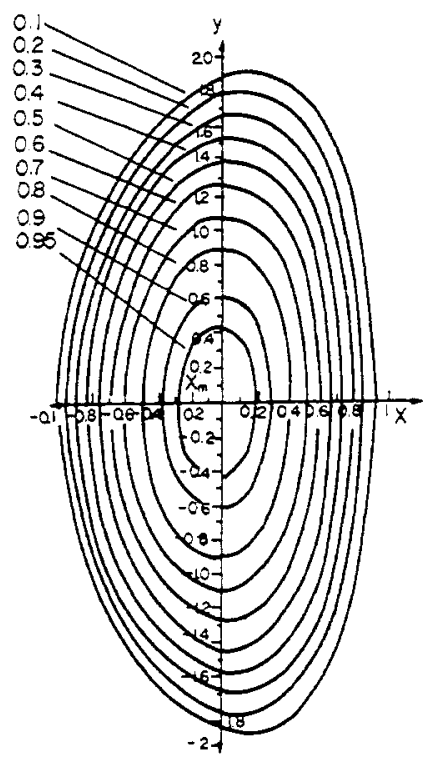

FIG. 4(b).-Magnetic surfaces in the plasma for $1 / q_{0}=0.18, \varepsilon_{s}=0.1, E=0.6, \varepsilon_{0}=0.1$, $\varepsilon_{\mathrm{k}}=0.1, \mu_{\mathrm{k}}=1, k=2, \alpha_{0}=0.024, \beta=0.07$ and different values of $F / F_{0}$. The magnetic axis is placed at distance $x_{m}=-0.05$ from the central axis of the plasma. 
and

$$
P_{0}^{\prime}=\frac{\sqrt{ }\left(1-E_{m}^{2}\right)}{\iota_{c 0}}\left(\frac{j_{s 0}}{2 B_{s 0}}+\frac{d^{\prime}(s)}{2}\right) \frac{\mathrm{d} P_{(F)}}{\mathrm{d} F}
$$

where $W=2 \varepsilon_{k}\left(x_{m} / a\right)-\left(\varepsilon_{k}{ }^{2}+\varepsilon_{m}{ }^{2}\right)\left(x_{m} / a\right)^{2}, x_{m} / a=\left[1-\sqrt{\left(1+3 \nu^{2}\right)}\right] / 3 \nu$ and the point $\left(x_{m}, 0\right)$ is assumed to be the position of the magnetic axis. The ellipticity $E_{m}$ of the magnetic surfaces near the m.a. and the functions $\tilde{r}, \tilde{s}$ which give the corrections to the elliptical form of the magnetic surfaces near the m.a. relative to the principle normal to the m.a. are given by:

$$
\begin{gathered}
E_{m}=\text { th } \eta=\frac{M_{1}(1+E)-M_{2}(1-E)}{M_{1}(1+E)+M_{2}(1-E)} \\
\sqrt{B_{s} \tilde{r}}=\frac{1}{2 a}\left(\frac{M_{2} N_{2}-M_{1} N_{1}}{M_{1} M_{2}}\right) \frac{\sqrt{ }\left(1-E_{m}^{2}\right)}{\left(2+E_{m}\right)}
\end{gathered}
$$

and

$$
\sqrt{B}_{s 0} \tilde{S}=0
$$

where

$$
\begin{aligned}
& M_{1}=\left(\gamma_{1} \varepsilon_{k} \nu-1\right)+3\left(\nu-\varepsilon_{s}\right)\left(\frac{x_{m}}{a}\right)+6 \nu\left(\varepsilon_{s}-\gamma_{1} \varepsilon_{k}\right)\left(\frac{x_{m}}{a}\right)^{2} \\
& M_{2}=\left(\gamma_{2} \varepsilon_{k} \nu-1\right)+\left(\nu+3 \varepsilon_{s}\right)\left(\frac{x_{m}}{a}\right)-\nu\left[3 \varepsilon_{s}+\varepsilon_{k}\left(\gamma_{1}+\gamma_{2}\right)\right]\left(\frac{x_{m}}{a}\right)^{2} \\
& N_{1}=\left(\nu+3 \varepsilon_{s}\right)-2 \nu\left[3 \varepsilon_{s}+\varepsilon_{k}\left(\gamma_{1}+\gamma_{2}\right)\right]\left(\frac{x_{m}}{\alpha}\right) \\
& N_{2}=\left(\nu-\varepsilon_{s}\right)+4 \nu\left(\varepsilon_{s}-\gamma_{1} \varepsilon_{k}\right)\left(\frac{x_{m}}{\alpha}\right) .
\end{aligned}
$$

The vacuum magnetic well $V_{0}$ " near the m.a. for our class of equilibria is given by:

$$
V_{0}^{\prime \prime}=-\frac{L}{\pi B_{\mathrm{s} 0}^{2} \sqrt{\left(1-E_{1 n}^{2}\right)}} Q_{0}^{\prime \prime}
$$

with

$$
Q_{0}^{\prime \prime}=\frac{a_{0}^{2}}{2}\left[1+2 \mu_{k}^{2}\left(2-E_{m}\right)\right]-\left(\frac{E_{m}}{2} \varepsilon_{m}\right)^{2}-\left[\left(2-E_{m}\right) C_{1}-3 E_{m} C_{2}\right] a_{0} \mu_{k}
$$

where

$$
C_{1}=\frac{1}{2 a} \frac{3 N_{2}(1+E)+N_{1}(1-E)}{3 M_{1}(1+E)}
$$

and

$$
C_{2}=\frac{1}{2 a} \frac{N_{2}(1+E)-N_{1}(1-E)}{2 M_{1}(1+E)}
$$


The investigation of the expression (20) shows that it is possible to have a system with vacuum magnetic well $V_{0}^{\prime \prime}<0$ with all different magnetic surfaces, and by allowing current to flow only in the forward direction (defined by $\left(1 / q_{0}\right)>(-k)$ or $G<0)$. We have a deeper magnetic well with horizontal elliptical $\left(E<0, \varepsilon_{s}=0\right)$ and triangular deformation $\left(E<0, \varepsilon_{s}<0\right)$ cross sections with an increase of the depth of modulation $\mu_{k}$, and a decrease of the number of modulation periods $k$.

\section{STABILITY}

The final form of Mercier's criterion near the m.a. is given by formula (70) of reference (MERCIER, 1964). This criterion for the class of equilibria described by the parameters $E_{m}=($ th $\eta(s)), j_{s 0}, B_{s 0}, d^{\prime}(s) / 2, \tilde{r}(s)$, and $\tilde{S}(s)$ being constants and the torsion of the m.a. $1 / T(s)=0$, has the form:

$$
\begin{aligned}
& \left(-P_{0}^{\prime}\right)\left[-\left(\frac{j_{s 0}}{2 B_{s 0}}\right)^{2} \frac{1}{\operatorname{ch}^{2} \eta}-\operatorname{th}^{2} \eta\left(\frac{d^{\prime}(s)}{2}\right)^{2}+\left(\text { th } \eta\left\langle\frac{\cos d(s)}{R_{(s)}^{2}}\right\rangle-\left\langle\frac{1}{R_{(s)}^{2}}\right\rangle\right)\right. \\
& +3 \text { th } \eta\left(\sqrt{ } B_{s 0} \tilde{r} e^{-\eta}\left\langle\frac{\cos d / 2}{R(s)}\right\rangle+\sqrt{ } B_{s 0} \tilde{S} e^{+\eta}\left\langle\frac{\sin d / 2}{R(s)}\right\rangle\right) \\
& \left.+\frac{B_{\mathrm{s} 0}{ }^{2}}{L \operatorname{ch} \eta} \operatorname{Re} \oint\left(\frac{P Z}{4 P_{0}^{\prime}}\right) \mathrm{d} s\right]>0
\end{aligned}
$$

where the average is taken over the length of the magnetic axis, i.e.

$$
\langle g(s)\rangle=\frac{1}{L} \int_{0}^{L} \mathrm{~d} s g(s),
$$

the prime (') means the derivatives with respect to $s, \mathscr{P}=\mathscr{P}_{1}-i \mathscr{P}_{2}, Z(s)=$ $\mathscr{H}_{1}(s)+i \mathscr{K}_{2}(s)$ and the functions $\mathscr{H}_{1}(s)$ and $\mathscr{H}_{2}(s)$ are the solutions of the couple of equations:

$$
\begin{aligned}
& \mathscr{H}_{1}^{\prime}(s)+\mathscr{K} \mathscr{H}_{2}(s)-\left(\frac{4 P_{0}^{\prime}}{B_{s 0}^{3 / 2}}\right) \mathrm{e}^{-n / 2}\left(\frac{\cos d / 2}{R(s)}\right)=0 \\
& \mathscr{H}_{2}^{\prime}(s)-\mathscr{K} \mathscr{H}_{1}(s)-\left(\frac{4 P_{0}^{\prime}}{B_{s 0}^{3 / 2}}\right) \mathrm{e}^{-n / 2}\left(\frac{\sin d / 2}{R(s)}\right)=0 .
\end{aligned}
$$

$\mathscr{P}_{1}$ and $\mathscr{P}_{2}$ for this class of equilibria are given by:

$$
\begin{aligned}
& \mathscr{P}_{1}=\frac{\left(2 / \sqrt{B_{s 0}}\right) e^{\eta / 2}}{(2 \operatorname{ch} \eta-\operatorname{sh} \eta)}\{\left\{\left(\frac{j_{s 0}}{B_{s 0}}\right)\left(\frac{\sin d / 2}{R(s)}\right)+\frac{\left|\iota_{c 0}\right|\left(\mathscr{H}_{1} v \overline{B_{s 0}}\right) s h^{2} \eta / 2}{4 \operatorname{ch} \eta / 2}\right. \\
&\left.+\frac{\operatorname{sh} \eta \mathrm{e}^{-\eta}}{4}\left(\frac{\cos d(s) / 2}{R(s)}\right)^{\prime}+\frac{5}{4} \operatorname{th} \eta\left(\frac{j_{s 0}}{2 B_{s 0}}+\frac{d^{\prime}(s)}{2}\right)\left(\frac{\sin d / 2}{R(s)}\right)\right\} \\
& \mathscr{P}_{2}=\frac{\left(2 / \sqrt{B_{s 0}}\right) \mathrm{e}^{-\pi / 2}}{(2 \operatorname{ch} \eta+\operatorname{sh} \eta)}\left\{\left(\frac{j_{s 0}}{B_{s 0}}\right)\left(\frac{\cos d / 2}{R(s)}\right)+\frac{\left|\iota_{c 0}\right|\left(\mathscr{H}_{2 \sqrt{ }} \overline{B_{s 0}}\right) \operatorname{sh}^{2} \eta / 2}{4 \operatorname{ch} \eta / 2}\right. \\
&\left.+\left(\frac{\operatorname{sh} \eta \mathrm{e}^{\eta}}{4}\right)\left(\frac{\sin d / 2}{R(s)}\right)^{\prime}+\frac{5}{4} \operatorname{th} \eta\left(\frac{j_{s 0}}{2 B_{s 0}}+\frac{d^{\prime}(s)}{2}\right)\left(\frac{\cos d / 2}{R(s)}\right)\right\} .
\end{aligned}
$$

It will be interesting to consider Mercier's criterion (21) for the real plasma configuration of modulated curvature $m$.a. without the rotation of the magnetic 
surfaces. This will enable us to compare the domains obtained with and without this helical images hypothesis. This criterion for the considered case $\left(d^{\prime}(s) / 2=0\right)$ has the form:

$$
\begin{aligned}
\left(-P_{0}{ }^{\prime}\right)[ & -\frac{1}{\operatorname{ch}^{2} \eta}\left(\frac{j_{s 0}}{2 B_{s 0}}\right)^{2}-\left\langle\frac{1}{R^{2}(s)}\right\rangle(1-\text { th } \eta)+3 \sqrt{B_{s 0}} \tilde{r} e^{-\eta} \text { th } \eta\left\langle\frac{1}{R(s)}\right\rangle \\
& \left.+\frac{2(1-\text { th } \eta)}{(2+\text { th } \eta)}\left(2+\frac{5}{4} \text { th } \eta\right)\left\langle\frac{1}{R^{2}(s)}\right\rangle+\left(\frac{P_{0}^{\prime}\left|c_{c 0}\right|}{B_{s 0}}\right) \frac{\mathscr{F}(\eta)}{2\left(j_{s 0} / 2 B_{s 0}\right)^{2}}\left\langle\frac{1}{R^{2}(s}\right\rangle\right]>0
\end{aligned}
$$

where

$$
\begin{aligned}
& \mathscr{F}(\eta)=\frac{4 \mathrm{e}^{-3 \eta / 2} \operatorname{sh}^{2}(\eta / 2) \operatorname{ch} \eta}{\operatorname{ch}(\eta / 2)(2 \operatorname{ch} \eta+\operatorname{sh} \eta)} \\
& =\frac{4 E_{m}{ }^{2} \sqrt{ }\left(1-E_{m}{ }^{2}\right)}{\left.\left(1+E_{m}\right)\left(2+E_{m}\right)^{2}\left[1+\sqrt{\left(1-E_{m}{ }^{2}\right.}\right)-\frac{E_{m}{ }^{2}}{\left(2+E_{m}\right)}\right]} .
\end{aligned}
$$

Condition (25) with $E_{m}=0$ is equivalent to that given by formula (26) in MERCIER (1963), and does not depend on the plasma pressure $P_{0}{ }^{\prime}$ term.

Figures (5) and (6) show the plots of $L j_{s 0} / 4 \pi B_{s 0}$ vs $\left|i_{c 0}\right| P_{0}^{\prime} / B_{s 0}$ for the domains of stability with vertical elliptical $\left(E_{m}=0.6, \sqrt{B_{s 0}} \tilde{r}=0\right)$ and triangular deformation $\left(E_{m}=0.6, \sqrt{B}_{s 0} \tilde{r}=0.1\right)$ plasma cross sections near the m.a. respectively. The domains of stability for a real plasma configuration are given by the dotted curves and are calculated from the condition (25) with the expression (1). These domains have a perfect symmetry between $k$ and $-k$ (without this rotation related to $k$ ) and will not change if the longitudinal current is inversed (i.e. $j_{s 0} \rightarrow-j_{s 0}$ ). The limiting value of $\beta$ for stability in this case becomes zero if $L j_{s 0} / 4 \pi B_{s 0}=0$.

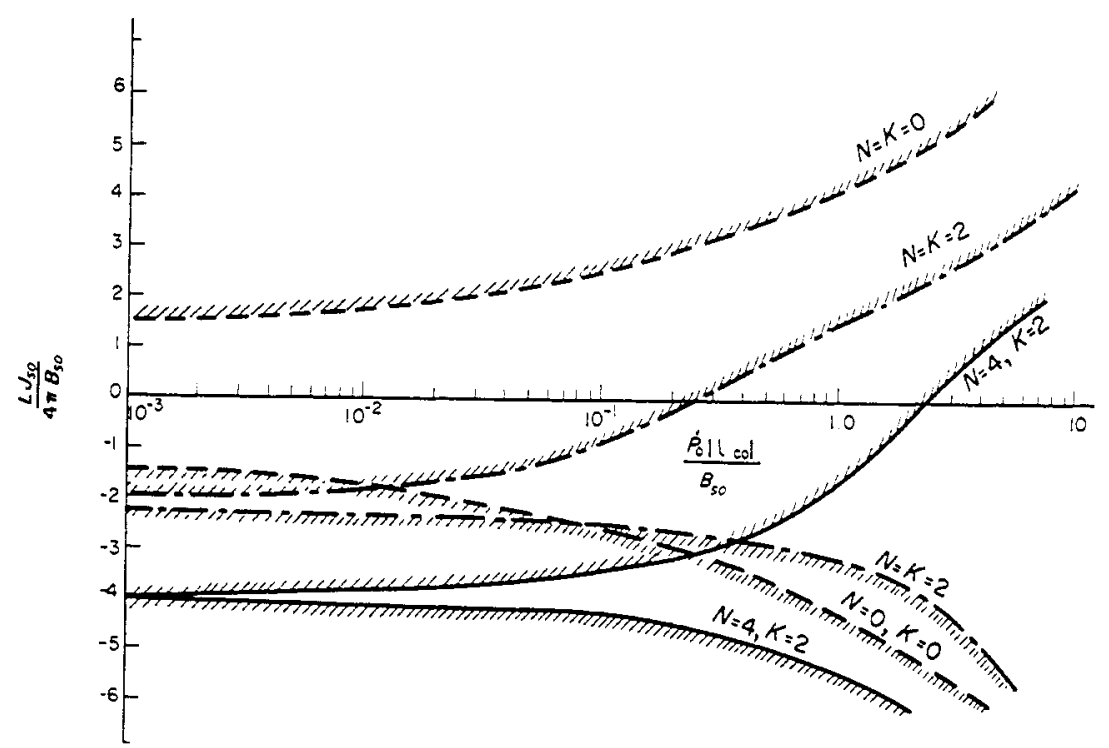

Fia. 5.-Comparison between the domains of stability with vertical elliptical plasma cross section near the magnetic axis for $E_{m}=0.6, \sqrt{ } \beta_{s} 0^{f}=0, \alpha_{0}=0.0233$, and $\mu_{k}=1$. 


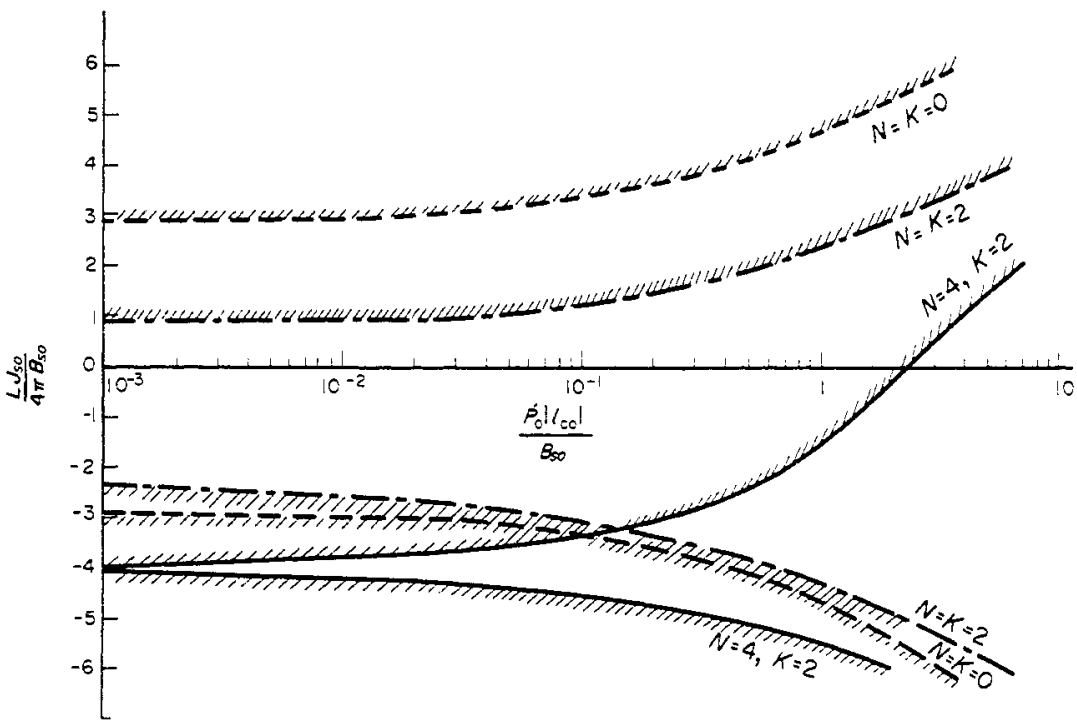

FIG. 6.-Comparison between the domains of stability with triangular deformation plasma cross section near the magnetic axis for $E_{m}=0.6 . \sqrt{ } B_{s 0} r=0.1, a_{0}=0.0233$, and $\mu_{k}=1$.

For the case in which the rotation of the magnetic surfaces has the form:

$$
\frac{d(s)}{2}=\frac{2 \pi N S}{L}
$$

where $N$ is an integer. The criterion of stability (21) corresponding to the resonance case $L \mathscr{K} / 2 \pi \ll 1$ is given by;

$$
\begin{aligned}
& \left(-P_{0}{ }^{\prime}\right)\left[-\frac{1}{\operatorname{ch}^{2} \eta}\left(\frac{j_{s 0}}{2 B_{\mathrm{s} 0}}\right)^{2}-\operatorname{th}^{2} \eta\left(\frac{\mathrm{d}^{\prime}(s)}{2}\right)^{2}+\left(\operatorname{th} \eta\left\langle\frac{\cos d(s)}{R^{2}(s)}\right\rangle-\left\langle\frac{1}{\left.R^{2}(s)\right)}\right\rangle\right)\right. \\
& +3 \text { th } \eta\left({ } B_{s 0} \tilde{r} \mathrm{e}^{-n}\left\langle\frac{\cos d / 2}{R(s)}\right\rangle+\sqrt{B_{s 0}} \tilde{S} \mathrm{e}^{n}\left\langle\frac{\sin d / 2}{R(s)}\right\rangle\right) \\
& \left.+\frac{B_{s 0}{ }^{2}}{L \operatorname{ch} \eta} \operatorname{Re} \oint\left(\frac{\mathscr{P} Z}{4 P_{0}^{\prime}}\right) d s\right]>0
\end{aligned}
$$

with

$$
\begin{aligned}
\frac{B_{s 0}{ }^{2}}{L \operatorname{ch} \eta} \oint & \left(\frac{\mathscr{P} Z}{4 P_{0}{ }^{\prime}}\right) \mathrm{d} s=\frac{4 a_{k}(1-\text { th } \eta)}{\left(\frac{j_{s 0}}{2 B_{s 0}}+\frac{d^{\prime}(s)}{2}\right)(2+\text { th } \eta)}\left\{\frac{j_{s 0}}{2 B_{s 0}}+\frac{5}{8}\left(\frac{j_{s 0}}{2 B_{s 0}}+\frac{d^{\prime}(s)}{2}\right) \text { th } \eta\right\} \\
& \times\left\langle\frac{\cos d / 2}{R(s)}\right\rangle+\left(\frac{\iota_{c 0} P_{0}{ }^{\prime}}{B_{s 0}}\right) \frac{a_{k}{ }^{2}}{\left(\frac{j_{s 0}}{2 B_{s 0}}+\frac{d^{\prime}(s)}{2}\right)^{2}} \frac{\mathscr{F}\left(E_{m}\right)}{2} .
\end{aligned}
$$

Condition (27) represents the criterion of stability in a geometrical plasma configuration model. The domains of stability given in Figs. 5 and 6 represented by 
the broken and solid curves are calculated from condition (27) with expression (1) for $N=k$ and $N \neq k$ respectively. The influence of the rotation $d^{\prime}(s) / 2(=$ $2 \pi N / L$ ) of the magnetic surfaces (which is associated with the helical hypothesis) casued a disturbance to the domains of stability and is symmetry when the longitudinal current is inversed. The limits of these domains in this case are given by the condition: $N^{2} \leq\left(L j_{s 0} / 4 \pi B_{s 0}\right)^{2} \leq N^{2} / \delta_{N}$ where $\delta_{N}$ is a numerical factor less than one. Kruskal limit is exceeded within this condition (the case $N=1$ corresponds to Kruskal limit). Indeed, the magnetic axis of the plasma configuration could become strongly inadequate for a plasma machine. The limiting value of $\beta$ for stability in the geometrical model plasma configuration becomes zero if $L j_{s 0} / 4 \pi B_{s 0}=-N$.

The final form of criterion (27) for the toroidal plasma configuration with an arbitrary rotation of $d^{\prime}(s) / 2(=2 \pi k / L)$ related to the $k$ Fourier coefficient of the development of the m.a. curvature which is given by expression (1) can be written as:

$$
\begin{aligned}
& \left(-P_{0}^{\prime}\right)\left[\left(E_{m}{ }^{2}-1\right)\left(\frac{j_{s 0}}{2 B_{s 0}}\right)^{2}-\left(k \alpha_{0} E_{m}\right)^{2}+a_{0}{ }^{2}\left\{\mu_{k}{ }^{2}\left(E_{m}-2\right)-1\right\}\right. \\
& +3 \sqrt{B_{s}} \tilde{r}\left(a_{0} \mu_{k}\right) E_{m} \sqrt{\left(\frac{1-E_{m}}{1+E_{m}}\right)}+\frac{2 a_{0}{ }^{2} \mu_{k}{ }^{2}}{\left(\frac{j_{s 0}}{2 B_{s 0}}+a_{0} k\right)^{2}}\left(\frac{1-E_{m}}{1+E_{m}}\right) \\
& \left.\times\left\{\frac{j_{s 0}}{2 B_{s 0}}\left(2+\frac{5}{4} E_{m}\right)+\frac{5}{4} k a_{0} E_{m}\right\}+\frac{2 a_{0}^{2} \mu_{k}{ }^{2}\left|\iota_{c 0}\right|}{B_{s 0}\left(\frac{j_{s 0}}{2 B_{s 0}}+k a_{0}\right)}\left(\frac{\mathrm{d} P}{\mathrm{~d} F}\right) \frac{\sqrt{\left(1-E_{m}\right)}}{4} \mathscr{F}\left(E_{m}\right)\right]>0 .
\end{aligned}
$$

The explicit expression for Mercier's criterion (29) for the class of equilibria described in the equilibrium solution can be determined by substituting respectively for the parameters $j_{s o} / 2 B_{s u}, \iota_{c 0} / 2 \pi, E_{m}, \tilde{r}$ from formulae (14)-(18). At high value of beta $\left(\beta^{*} \sim O(1 / \varepsilon)\right)$ the expressions for these parameters with all magnetic surfaces (circular or non-circular) depend on the plasma pressure. In other words, the effect of the plasma pressure on the criterion of stability (29) is not linear with respect to $\beta^{*}$. It is not easy to determine analytically to what order of $\beta^{*}$ the plasma pressure has an effect on this criterion. At low beta $\left(\beta^{*} \leqslant 1\right)$ we find that $\quad x_{m} / a \sim \varepsilon, \quad \nu \sim \varepsilon, \quad E_{m} \sim E, \quad M_{1} \simeq M_{2} \simeq-1, \quad N_{1} \simeq \nu+3 \varepsilon_{s}, \quad N_{2} \simeq \nu-\varepsilon_{s}$. $\left[\left(M_{2} N_{2}-M_{1} N_{1}\right) / M_{1} M_{2}\right] \sim 4 \varepsilon_{s}, W \simeq 0, j_{s 0} \sim j_{0}, B_{s 0} \sim B_{0}$ and

$$
\left(P_{0}^{\prime} / B_{0}\right)=-\frac{2 \sqrt{\left(1-E_{m}{ }^{2}\right)}}{\iota_{c 0}} \beta *\left(\frac{\varepsilon_{0} G}{a}\right)^{2} .
$$

In this case condition (29) takes the form:

$$
\begin{aligned}
\left(-P_{0}^{\prime}\right)\left[\left(\frac{1}{q_{0}}\right)^{2}\right. & \left(E^{2}-1\right)-(k E)^{2}+\left(\mu_{k}^{2}(E-2)-1\right)+\left(\frac{6 \varepsilon_{s} \mu_{k}}{\varepsilon_{0}}\right)\left(\frac{1-E}{1+E}\right) \\
& \left.\left.-\left(\frac{4 \mu_{k}^{2}}{G}\right)\left(\frac{1-E}{1+E}\right)\left(\frac{1}{q_{0}}-\frac{5}{8} E G\right)-\mu_{k}^{2}\left(\frac{\left|\iota_{c 0}\right|}{\iota_{c 0}}\right) \beta^{*} \sqrt{\left(1-E^{2}\right.}\right) \mathscr{F}(E)\right]>0 .
\end{aligned}
$$




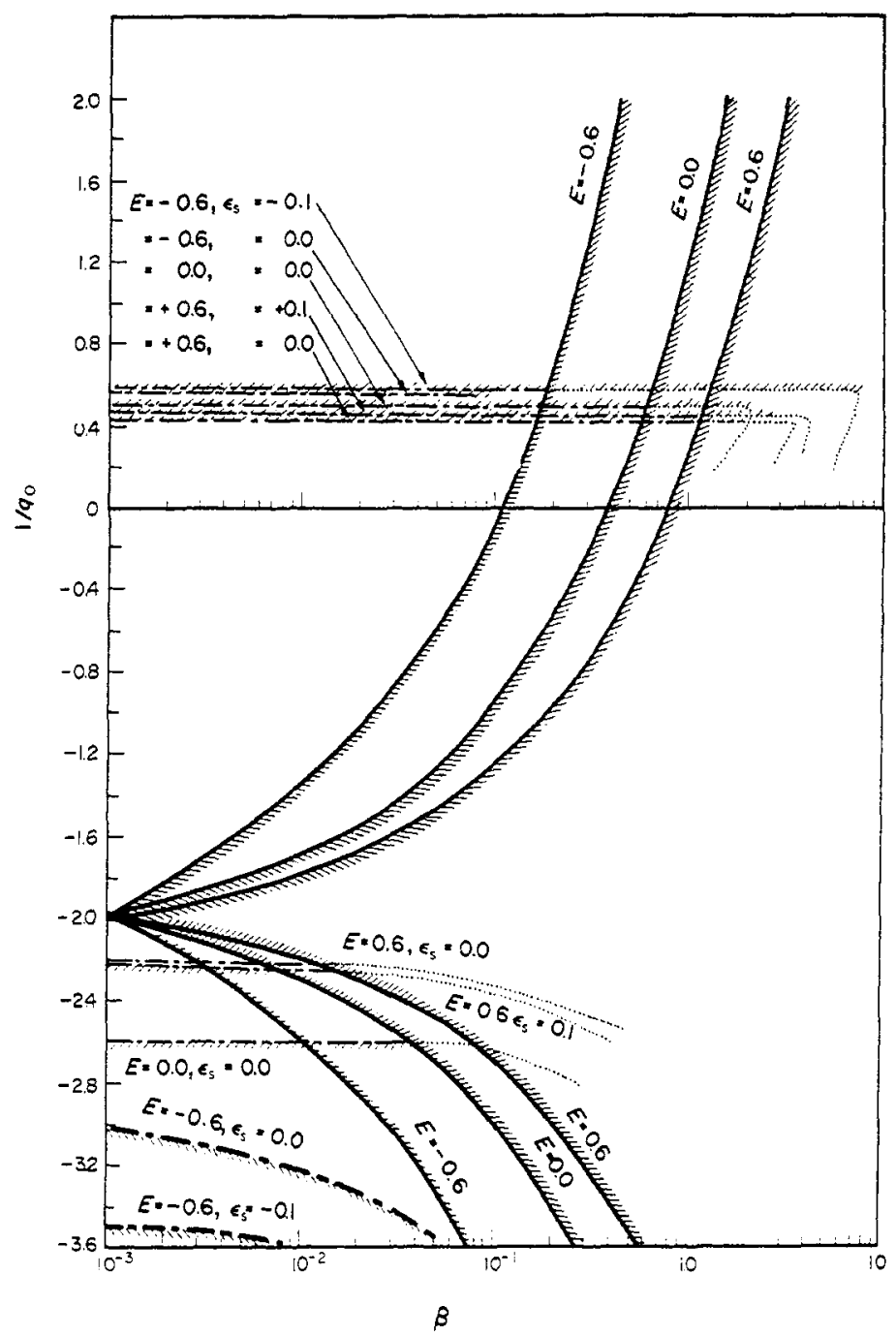

FIG. 7.-Domains of equilibrium and stability for the considered toroidal plasma configuration characterized by $k=2, \mu_{k}=1, \varepsilon_{0}=0.1, \alpha=4.291 \mathrm{~S}, \alpha_{0}=0.0233$, and different values of $E$ and $\varepsilon_{\mathrm{s}}$.

It is clear that the effect of the plasma pressure on the condition (30) is linear with respect to $\beta^{*}$ and vanishes with that of the circular cross section.

The numerical calculations represented by Figs. 7 and 8 show the plot of $1 / q_{0}$ vs the limiting value of $\beta$ for equilibrium calculated from equation (13). These are shown by the solid curves while those for stability as calculated from (29) are indicated by the broken curves. The intersection of curves limiting the domain of equilibrium and stability gives the limiting value of $\beta$ for each magnetic surface. We observe that the domains for all the equilibrium and stability depend on the direction of the longitudinal current $I_{s}$. Figures 7 and 8 show respectively the influence of the non-circular cross section and the number of modulations with their depths on the domain of equilibrium and stability. 


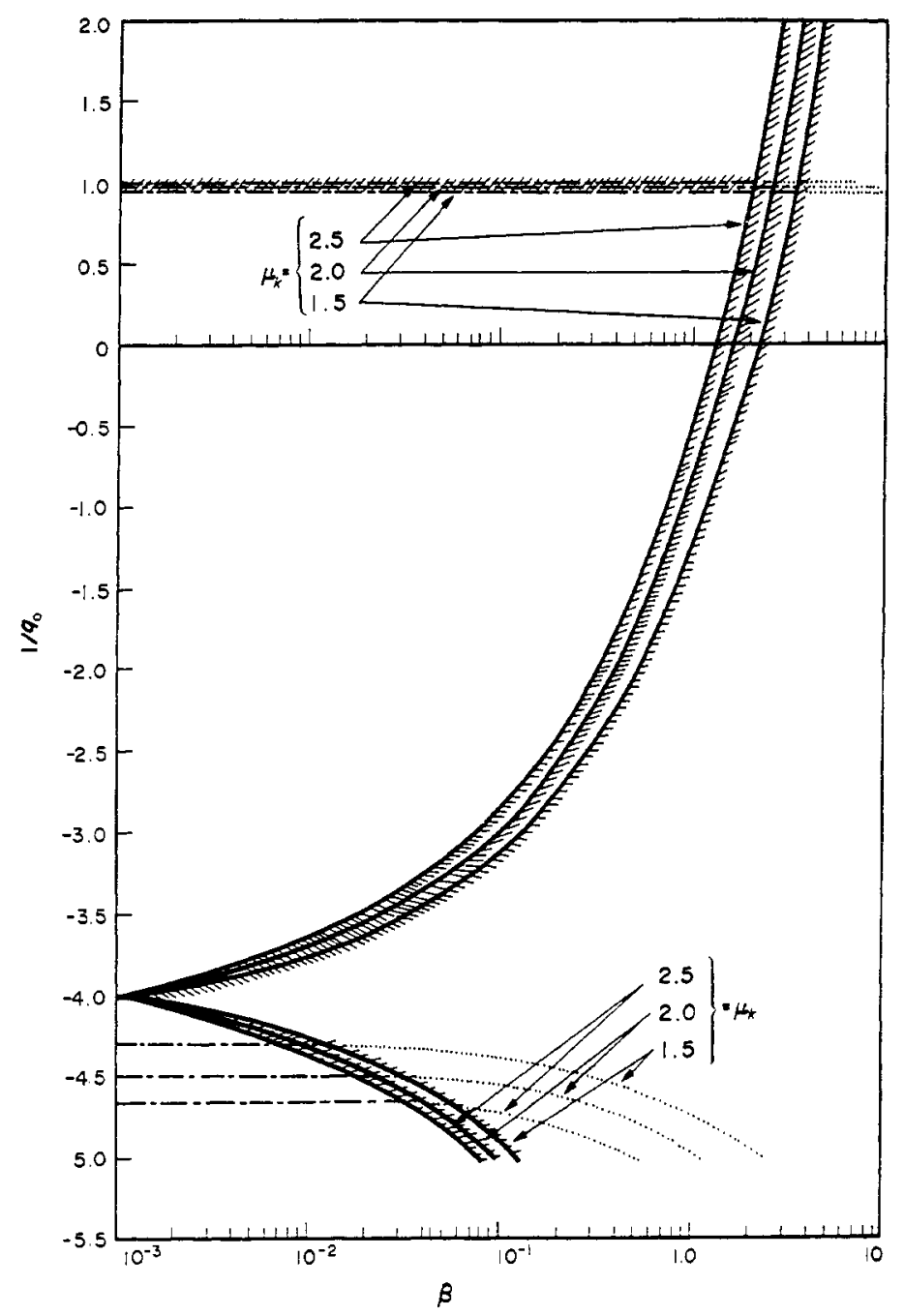

FIG. 8.-Domains of equilibrium and stability for the considered toroidal plasma configuration with vertical elliptical cross section characterized by $E=0.6, \varepsilon_{s}=0$, $\varepsilon_{0}=0.1, \alpha=4.2918, \alpha_{0}=0.0233, K=4$, and different values of $\mu_{k}$.

As it is clear from equation (13) that the case $1 / q_{0}=-k_{0}$ corresponds to the resonance case at which $G=0$ and $\beta=0$. We note that the influence of triangular deformation on the limiting value of beta for equilibrium is weak and can be neglected. A vertically elongated elliptic $\left(E>0, \varepsilon_{s}=0\right)$ plasma cross section is preferable for equilibrium. The limiting value of beta for equilibrium increases with the increasing of the number of modulation periods and the decreasing of the depth of modulation.

The domains of stability exist with all possible shapes of the magnetic surfaces. It increases in the forward direction due to the appearance of the magnetic well $\left(V_{0}{ }^{\prime \prime}<0\right)$. This domain decreases in the backward direction (defined by $1 / q_{0}<$ $\left(-k_{0}\right)$ or $\left.G>0\right)$. In both directions with $\left(\beta<\beta_{P_{\operatorname{mex}}}\right)$, the horizontal elliptical and triangular deformations are the most preferable plasma cross sections for stability 
(a deeper magnetic well exists with these cross sections). Also, the domains of stability increase by increasing the depth of modulation and decreasing the number of modulation periods.

\section{CONCLUSIONS}

The equilibrium and stability near an arbitrary modulated planar magnetic axis of a toroidal plasma configuration with non-circular cross sections are studied. This plasma configuration is a geometrical configuration model in which the magnetic surfaces arbitrarily rotate along the magnetic axis. From investigating the domains of stability in both real and geometrical model plasma configurations, it is found that these rotations of the magnetic surfaces cause a disturbance to the domains of stability and their symmetry. These domains are given the condition $N^{2} \leq\left(L j_{s 0} / 4 \pi B_{s 0}\right)^{2} \leq N^{2} / \delta_{N}$ where $N$ is the number of rotations of the magnetic surfaces and $\delta_{N}$ is a numerical factor less than one. Kruskal limit is exceeded within this condition.

Also, it is found from the domains of equilibrium and stability (which belong to the $k$-Fourier resonant coefficient of the development of the curvature of the magnetic axis) that the vertical elliptical plasma cross section with increasing the number of modulation periods and decreasing the depth of the modulation is preferable for equilibrium. On the other hand, a horizontal elliptical or triangular deformation plasma cross section with decreasing of the number of modulation periods and increasing the depth of modulation is preferable for stability.

At this point, one would like to mention qualitatively that the influence of the other neglected Fourier coefficient $\alpha_{0}$ on the stability of the plasma configuration under consideration may add a stabilization contribution within the above conditions, and otherwise add a de-stabilization contribution.

Acknowledgement-The author is indebted to Dr C. MERCIER of Association Euratom-CEA, France for helpful discussion and continued encouragement.

\section{REFERENCES}

Aburdzhanrya K. D. and Mixharlovski A. B. (1978) Sov. J. Plasma Phys. 4, 115.

ADAMS J. C. and Mercier C. (1969) Plasma Physics and Controlled Fusion Research. 1. 199. IAEA. Luc H., Mashke E. K., Mercier C.. Misguich H. and Rizk H. M. (1974) Plasma Physics 5, 455. MERCIER C. (1963) Nucl. Fusion 3, 89.

Mercier C. (1964) Nucl. Fusion 4, 213.

Mikhanovskit A. B. and Aburdzhaniya K. D. (1979) Plasma Physics 21, 109.

Shafranov V. D. (1968) Nucl. Fusion 8, 253. 